\title{
ANÁLISE DA FLEXIBILIDADE E DA AUTOESTIMA EM PARTICIPANTES DE UM PROGRAMA DE ORIENTAÇÃO E REEQUILÍBRIO POSTURAL EM GRUPO
}

Raíssa Puzzi Ladvig ${ }^{1}$, Cíntia Tiemi Gushiken², Fernanda Berno dos Santos ${ }^{2}$, Felipe Novaes Anadão ${ }^{2}$, Dalva Minonroze Albuquerque Ferreira ${ }^{3}$, Cristina Elena Prado Teles Fregonesi ${ }^{3}$, Célia Aparecida Stellutti Pachioni ${ }^{3}$, Camilla Leonel Santos ${ }^{4}$, Lisiane Yuri Sako ${ }^{4}$

Universidade Estadual Paulista - UNESP, ${ }^{1}$ Curso de Especialização em Ortopedia e Traumatologia, ${ }^{2}$ Curso de Graduação em Fisioterapia, ${ }^{3}$ Departamento de Fisioterapia, ${ }^{4}$ Residencia em Ortopedia e Traumatologia, Presidente Prudente, SP. e-mail: ladvigra@hotmail.com

\section{RESUMO}

O objetivo deste estudo foi avaliar a flexibilidade e a autoestima de participantes que iniciarão as atividades num programa de orientação e reequilíbrio postural em grupo. O estudo é uma análise descritiva da flexibilidade e da autoestima em doze pacientes adultos de ambos os gêneros na faixa etária de 25 a 65 anos. A avaliação da flexibilidade foi composta pelo teste do Terceiro Dedo-Chão e Banco de Wells (teste de Sentar e Alcançar), e para avaliação da autoestima foi utilizada a escala de autoestima de Rosenberg. O presente estudo mostrou que a flexibilidade e a autoestima avaliadas nos participantes do programa se apresentaram dentro dos parâmetros de normalidade, na maioria deles. A participação de uma atividade física em grupo aumenta a competitividade e a socialização entre os participantes, servindo de incentivo, e esses benefícios contribuem nas atividades funcionais e na melhora da autoestima.

Palavras-chave: postura, flexibilidade, autoestima, atividade física, consciência corporal.

\section{ANALYSIS OF FLEXIBILITY AND SELF-ESTEEM IN AN ORIENTATION PROGRAM PARTICIPANTS AND REBALANCING POSTURAL GROUP}

\begin{abstract}
The aim of this study was to evaluate the flexibility and self-esteem of participants will start the activities in the orientation program and postural rebalancing group. The study is a descriptive analysis of flexibility and self-esteem in twelve adult patients of both genders aged 25-65 years. The flexibility assessment was made by the test Finger ThirdFloor and Bank Wells (Sit and Reach test), and assessment of self-esteem we used the Rosenberg self-esteem scale. This study showed that flexibility and assessed self-esteem in the participants of the program were within normal limits in most of them. The participation of a physical activity group increased competitiveness and socialization among the participants, serving as incentive, and these benefits contribute in functional activities and improving selfesteem.
\end{abstract}

Keywords: posture, flexibility, self-esteem, physical activity, body awareness.

\section{INTRODUÇÃO}

A maior parte dos problemas com a nossa postura é resultado de efeitos acumulados de uma má postura, uma vida estressante, maus posicionamentos no trabalho, maus hábitos no dormir e levar uma vida sedentária. Pode-se dizer então, que os desequilíbrios são classificados como mecânicos, emocionais e orgânicos'. Os hábitos posturais estão intimamente ligados à limitação da amplitude das articulações, da extensibilidade dos músculos e da plasticidade dos ligamentos e tendões ${ }^{2}$.

A imagem corporal refere-se a um conjunto que engloba percepção, emoção, sentimentos e pensamentos direcionados ao próprio corpo, sendo a autoestima o conjunto desses sentimentos e pensamentos do indivíduo sobre seu próprio valor, competência e adequação, que repercute em atitude positiva ou negativa em relação a si. Evidências indicam relação positiva da autoestima negativa com a insatisfação corporal ${ }^{3}$.

A flexibilidade é considerada como um importante componente da aptidão física, relacionada à saúde e ao desempenho atlético, fazendo-se necessária também para realização de atividades de vida diária de qualidade. É muito importante, pois favorece uma maior mobilidade nas atividades diárias e esportivas, diminui o risco de lesões, favorece o aumento da qualidade e quantidade de movimentos e uma melhora da postura corporal ${ }^{4}$. 
Quando um músculo perde sua flexibilidade normal, ocorre uma alteração na relação comprimento-tensão, incapacitando-o de produzir um pico de tensão adequado, o que desenvolve fraqueza com retração muscular. 0 encurtamento muscular pode ser decorrente de diversos fatores, tais como alinhamento postural incorreto, imobilização do músculo, fraqueza muscular e envelhecimento ${ }^{5}$.

Um projeto de extensão de orientação e reequilíbrio postural em grupo, que vem proporcionar aos seus participantes, atividades físicas que atuam de forma global por meio de alongamentos, trabalho respiratório, consciência corporal, relaxamento, propriocepção e equilíbrio. À vista disso, o objetivo deste estudo foi avaliar a flexibilidade e a autoestima de participantes que iniciarão as atividades no "Programa de orientação e reequilíbrio postural em grupo" desenvolvido na Faculdade de Ciências e Tecnologia da UNESP - Campus de Presidente Prudente.

\section{MÉTODOLOGIA}

O estudo é uma análise descritiva da flexibilidade e da autoestima em doze pacientes adultos de ambos os gêneros na faixa etária de 25 a 65 anos aptos a realizarem atividade física e com encaminhamento médico, e que foram avaliadas para iniciarem a participação em um projeto de extensão universitária de orientação e reequilíbrio postural em grupo.

O Projeto de Extensão acontece num centro de estudos e de atendimentos em fisioterapia e reabilitação da Faculdade de Ciências e Tecnologia/UNESP, Campus de Presidente Prudente e foi aprovado e reconhecido pela Pró-Reitoria de Extensão Universitária da Universidade Estadual Paulista Júlio de Mesquita Filho - UNESP, e foi aprovado pelo Comitê de Ética em Pesquisa local com CAAE: 46648215.7.0000.5402, Número do Parecer: 1.152.961. Os indivíduos assinaram um Termo de Consentimento Livre e Esclarecido para participação na pesquisa. Os critérios de exclusão da pesquisa foram o uso de próteses e/ou órteses, cirurgias realizadas na coluna e gestação.

A avaliação da flexibilidade foi composta pelo teste de flexibilidade da cadeia posterior pela medida $(\mathrm{em} \mathrm{cm})$ da distância do dedo-chão durante a flexão anterior do tronco na posição ortostática, quanto mais se aproximar ou chegar a zero centímetros do chão maior a flexibilidade ${ }^{6}$, e o mesmo movimento foi avaliado $(\mathrm{em} \mathrm{cm})$ no banco de Wells, modelo instant flex, Sanny ${ }^{\circledR}$, Brasil, (teste de Sentar e Alcançar), sendo solicitada a flexão anterior da coluna vertebral com os membros inferiores em extensão, com a cabeça entre os braços também estendidos, mantendo-se estático a partir da posição de máximo alcance do movimento, sendo realizadas três tentativas nas quais se considera o melhor resultado, classificando a flexibilidade em excelente (igual ou acima de $22 \mathrm{~cm}$ ), boa (19 a $21 \mathrm{~cm})$, média $(14 \mathrm{a} 18 \mathrm{~cm})$, regular $(12 \mathrm{a} 13 \mathrm{~cm})$ e fraca (igual ou baixo de $11 \mathrm{~cm})^{7}$. Para avaliação da autoestima foi utilizada a escala de autoestima de Rosenberg que é uma medida unidimensional constituída por dez afirmações relacionadas a um conjunto de sentimentos de autoestima e auto aceitação que avalia a autoestima global. A metade dos itens está enunciada positivamente e a outra metade negativamente. Para cada afirmação existem quatro opções de resposta ( concordo totalmente $=4$, concordo $=3$, discordo $=2$ e discordo totalmente=1). É necessário realizar algumas inversões para obter a pontuação (itens $3,5,8,9$ e 10 ) e a média dos 10 itens dará a cotação da escala, cuja pontuação total oscila entre 10 e 40 . A obtenção de uma pontuação alta reflete uma autoestima elevada ${ }^{8}$.

\section{RESULTADOS}

Foram analisados 12 participantes (11 mulheres e um homem), com diagnóstico de: escoliose (6), lombalgia (4), hérnia de disco (1) e disfunção da articulação temporomandibular (ATM) (1), conforme apresentados na Tabela 1. 
Tabela 1. Análise descritiva da patologia, da idade (anos), da massa corporal $(\mathrm{kg})$, da estatura $(\mathrm{m})$ e do Índice de Massa Corpórea $(\mathrm{IMC})\left(\mathrm{Kg} / \mathrm{m}^{2}\right)$ acompanhados da média (M) e desvio padrão (DP) dos 12 participantes.

\begin{tabular}{|c|c|c|c|c|c|}
\hline Partic. & Patologia & Idade & Massa & Estatura & IMC \\
\hline 1 & Lombalgia & 59 & 76 & 1,48 & 34,69 \\
\hline 2 & Escoliose & 29 & 53 & 1,59 & 20,96 \\
\hline 3 & Escoliose & 39 & 53 & 1,61 & 20,44 \\
\hline 4 & Escoliose & 60 & 64 & 1,56 & 26,29 \\
\hline 5 & Lombalgia & 59 & 64 & 1,56 & 26,29 \\
\hline 6 & Escoliose & 61 & 63 & 1,62 & 24,00 \\
\hline 7 & Escoliose & 63 & 78 & 1,73 & 26,06 \\
\hline 8 & Lombalgia & 62 & 60 & 1,53 & 25,63 \\
\hline 9 & Hérnia de disco & 51 & 69 & 1,60 & 26,95 \\
\hline 10 & Escoliose & 60 & 48 & 1,50 & 21,33 \\
\hline 11 & Disfunção de ATM & 23 & 55 & 1,58 & 22,03 \\
\hline 12 & Lombalgia & 23 & 61 & 1,61 & 23,53 \\
\hline$M \pm D P$ & & $49,08 \pm 15,97$ & $62,0 \pm 9,15$ & $1,58 \pm 0,06$ & $24,85 \pm 3,87$ \\
\hline
\end{tabular}

ATM - Articulação Temporomandibular

A tabela 2 mostra o resultado da avaliação da flexibilidade da cadeia posterior dos 12 participantes pelo teste do Terceiro DedoChão e pela medida no Banco de Wells.

$\mathrm{Na}$ avaliação da autoestima pela escala de Rosenberg na tabela 3, verificou-se que média dos participantes foi de 34,66 pontos, sendo que a menor pontuação foi de 25 pontos.
Tabela 2. Valores individuais dos testes de flexibilidade (cm): Terceiro Dedo-Chão (TDC) e as 3 medidas (a melhor em negrito) do Banco de Wells (BW) acompanhados da média (M) e desvio padrão (DP) nos 12 participantes.

\begin{tabular}{ccc}
\hline Partic. & TDC & $\begin{array}{c}\text { BW medidas } \\
\mathbf{1 / 2 / 3}\end{array}$ \\
\hline $\mathbf{1}$ & 17 & $\mathbf{2 2} / 21 / 21,5$ \\
$\mathbf{2}$ & 0 & $\mathbf{3 4} / 32,5 / 33,5$ \\
$\mathbf{3}$ & 30 & $4 / 7,5 / \mathbf{8 , 5}$ \\
$\mathbf{4}$ & 23 & $14 / \mathbf{1 5} / 12$ \\
$\mathbf{5}$ & 0 & $40 / 41 / \mathbf{4 2}$ \\
$\mathbf{6}$ & 0 & $34 / 35 / \mathbf{3 5}$ \\
$\mathbf{7}$ & 0 & $34 / 36 / \mathbf{3 7}$ \\
$\mathbf{8}$ & 20 & $23,5 / 26 / \mathbf{2 6 , 5}$ \\
$\mathbf{9}$ & 19 & $29 / 24 / \mathbf{3 1}$ \\
$\mathbf{1 0}$ & 0 & $41,5 / \mathbf{4 2} / \mathbf{4 1 , 5}$ \\
$\mathbf{1 1}$ & 8 & $27 / 27 / \mathbf{2 7 , 5}$ \\
$\mathbf{1 2}$ & 23 & $11 / 15 / \mathbf{1 6}$ \\
$\mathbf{M} \pm$ DP & $\mathbf{1 1 , 6 6} \pm$ & $\mathbf{2 8 , 0 4} \pm \mathbf{1 0 , 0 8}$ \\
& $\mathbf{1 1 , 4 3}$ & \\
\hline
\end{tabular}

TDC - Terceiro Dedo-Chão

BW - Banco de Wells 
Tabela 3. Valores individuais da pontuação da Escala de Autoestima de Rosenberg acompanhados da média (M) e desvio padrão (DP) nos 12 participantes.

\begin{tabular}{|c|c|c|c|c|c|c|c|c|c|c|c|c|c|}
\hline Partic. & 1 & 2 & 3 & 4 & 5 & 6 & 7 & 8 & 9 & 10 & 11 & 12 & $M \pm D P$ \\
\hline Autoestima & 31 & 32 & 25 & 36 & 39 & 39 & 39 & 39 & 38 & 38 & 28 & 32 & $34,66 \pm 4,90$ \\
\hline
\end{tabular}

\section{DISCUSSÃO}

Os resultados obtidos por meio dos testes de flexibilidade apresentaram grandes variações entre os participantes, onde a flexibilidade variou de normal $(0 \mathrm{~cm}$ em 5 participantes) para muito encurtamento no teste do Terceiro Dedo-Chão (20 a $30 \mathrm{~cm}$ em 4 participantes) e no Banco de Wells variou de excelente (igual ou acima de 22 $\mathrm{cm}$ em 9 participantes) a média (entre 14 e $18 \mathrm{~cm}$ em 2 participantes) e ainda fraca (igual ou baixo de $11 \mathrm{~cm}$ em um participante). A flexibilidade é uma variável cineantropométrica, que está relativamente associada à forma física, e é altamente treinável. Portanto, para manter uma postura adequada é necessário apresentar um mínimo de flexibilidade. Além disso, ela tem sido uma das qualidades físicas avaliadas como um componente fundamental no rastreamento do nível funcional do indivíduo, principalmente do idoso'.

A maior medida observada no teste do Terceiro Dedo-Chão, a menor medida no Banco de Wells e a menor pontuação na avaliação da autoestima, foram obtidas pela mesma participante (participante número 3). Pressupõese desde modo a relação corpo e mente, a falta de flexibilidade ocasionando a má postura, refletindo em uma imagem corporal ruim, acometendo dessa forma a autoestima do indivíduo. Estas medidas mostram que a manutenção de uma amplitude de movimento adequada das articulações é permitida pela flexibilidade onde os hábitos posturais podem ser determinados pela extensibilidade dos músculos e, consequentemente, pela limitação da amplitude $^{10}$.

Segundo o estudo de Dantas et al. ${ }^{11}$, a flexibilidade declina com a idade, os indivíduos sofrem uma perda de 20 a $30 \%$ entre 30 e 70 anos. E os parâmetros relativos à flexibilidade (mobilidade articular e elasticidade muscular) são funcionais em relação à respectiva faixa etária. No presente estudo a flexibilidade variou muito em relação à idade dos participantes, pois, 4 com mais de 50 anos (participantes 5, 6, 7 e 10) apresentaram os melhores valores de flexibilidade e dos três participantes com menos de 30 anos somente um apresentou valores satisfatórios de flexibilidade.

De acordo com Keleman ${ }^{12}$, a mente e o corpo funcionam em conjunto, é fundamental a relação entre personalidade e postura, pois o estado emocional pode ser uma das causas das alterações posturais, assim como a postura pode influenciar no estado emocional do individuo. Pela escala de Rosenberg verificamos que apenas dois participantes apresentaram, pela classificação, um valor médio de autoestima (participantes 3 e 11; valores de 25 e 28, respectivamente), porém quando analisamos a média do grupo, verifica-se que a autoestima pode ser considerada alta (acima de 30). Portanto, esses resultados não confirmam a presença de baixa autoestima nos participantes do estudo, fato que não confirmou a declaração de Keleman ${ }^{12}$ quando afirma que as alterações posturais podem influenciar no estado emocional.

Salve e Bankoff ${ }^{13}$ também constatam que um indivíduo com a autoestima elevada, tende a apresentar um padrão postural adequado, ao contrário do individuo que possui a autoestima baixa, ou sentimentos reprimidos de tristeza que os fazem apresentar uma postura retraída, estes tendem a ter a postura mais alterada como resposta da auto defesa do corpo, alterando a flexibilidade. Quanto a flexibilidade podemos sim afirmar que uma boa flexibilidade pode estar relacionada com uma autoestima elevada, fato esse observado na maioria dos participantes analisados.

A proposta de um programa de atividade física em grupo, é apresentada por Martins et al. $^{14}$, onde sua eficácia em pacientes com lombalgia crônica foram verificadas tanto nos aspectos físicos como nos mentais. 
Estes participantes foram avaliados para frequentarem um "Programa de orientação e reequilíbrio postural em grupo", pois, embora a flexibilidade e a autoestima estejam dentro dos valores de normalidade na maioria deles, o estudo de Korelo et al. ${ }^{15}$ mostrou que existem programas variados de cinesioterapia, e o que determina melhor condicionamento físico e, consequentemente, melhor qualidade de vida, é a persistência do indivíduo em querer obter os melhores resultados, através da conscientização de que ele próprio é gerenciador da sua saúde. Neste aspecto, esses pacientes e o programa proposto podem acrescentar vantagem quanto aos programas terapêuticos convencionais, pois são educativos, em grupo e incentivadores de melhores resultados quanto a flexibilidade e aspectos emocionais relacionados a sua patologia.

As limitações do estudo foram o baixo número amostral, pois a amostra foi por conveniência, ou seja, foram avaliados os pacientes que procuraram o centro de estudos e de atendimentos em fisioterapia e reabilitação com encaminhamento médico no período da coleta de dados, antes de iniciar as atividades em grupo. O estudo continuará coletando dados e realizará uma avaliação final que permitirá verificar a influência que o programa de orientação e reequilíbrio postural em grupo ocasionou nas medidas da flexibilidade e da autoestima.

\section{CONCLUSÃO}

O presente estudo mostrou que a flexibilidade e a autoestima avaliadas nos participantes do "Programa de orientação e reequilíbrio postural em grupo" se apresentaram dentro dos parâmetros de normalidade na maioria deles, porém, um participante mostrou valores mais baixos de flexibilidade e de autoestima, evidenciando haver uma relação dessas variáveis, onde os problemas posturais confirmados pelo diagnóstico dos participantes podem alterar $o$ aspecto emocional e a flexibilidade ou vice-versa. Portanto, a participação de uma atividade física em grupo aumentará a competitividade e a socialização entre os participantes, servindo de incentivo, e esses benefícios contribuirão nas atividades funcionais e na melhora da autoestima.

\section{CONFLITO DE INTERESSE}

Os autores declaram não haver qualquer potencial de conflito de interesse que possa interferir na imparcialidade deste trabalho científico.

\section{REFERÊNCIAS}

1. Verderi E. Programa de educação postural. 2.ed. São Paulo: Phorte; 2008.

2. Molinari B. Avaliação médica e física para atletas e praticantes de atividades físicas. São Paulo: Roca; 2000. 3. Fortesa LS, Ciprianib FM, Coelhob FD, Paesb ST, Ferreirab MEC. A autoestima afeta a insatisfação corporal em adolescentes do sexo feminino? Rev Paul Pediatr. 2014;32(3):236-40. DOI: http://dx.doi.org/10.1590/0103-0582201432314

4. Badaro AFV, Silva AH, Beche D. Flexibilidade versus Alongamento: esclarecendo as diferenças. Rev Saúde. 2007;33(1):32-6.

DOI: http://dx.doi.org/10592/22365834

5. Moreno A, Catai AM, Teodori RM, Borges BLA, Cesar $M C$, Silva E. Efeito de um programa de alongamento muscular pelo método de Reeducação Postural Global sobre a força muscular respiratória e a mobilidade toracoabdominal de homens jovens sedentários. J Bras Pneumol. 2007;33(6):679-86. DOI: http://dx.doi.org/10.1590/S1806-

37132007000600011

6. Mello JR. Análise comparativa da flexibilidade da coluna vertebral em acadêmicos de fisioterapia e educação física através do índice de Shober, índice de Stibor e teste do 30 dedo solo. [monografia]. Lins: Centro Católico Salesiano Auxilium, 2007.

7. Farias Jr. JC, Barros MVG. Flexibilidade e aptidão física relacionada à saúde. Corporis. 1998;3(1).

8. Hutz CS, Zanon C. Revisão da adaptação, validação e normatização da escala de autoestima de Rosenberg. Aval Psicol. 2011;10(1):41-9.

9. Netz Y, Argov E. Assessment of functional fitness among independent older adults: a preliminary report. Percept Mot Skills. 1997;84(3Pt1):1059-74. DOI: http://dx.doi.org/10.2466/pms.1997.84.3.1059

10. Veiga PHA, Daher CRDM, Morais MFF. Alterações posturais e flexibilidade da cadeia posterios nas lesões em atletas de futebol de campo. Rev Bras Ciênc Esporte. 2011;33(1):235-48. DOI: http://dx.doi.org/10.1590/S010132892011000100016

11. Dantas EHM, Pereira SAM, Aragão JC, Ota AH. A preponderância da diminuição da mobilidade articular ou da elasticidade muscular na perda da flexibilidade no envelhecimento. Fit Perf J. 2002;1(3):12-20. DOI: http://dx.doi.org/10.3900

12. Keleman S. Anatomia emocional. 5.ed. São Paulo: Summus; 1985.

13. Salve MGC; Bankoff ADP. Postura corporal - um problema que aflige os trabalhadores. Rev Bras Saúde 
Ocup. 2003;28(105/106):91-103. DOI: http://dx.doi.org/10.1590/S0303-

76572003000100010

14. Martins MRI et al. A eficácia da conduta do Grupo de Postura em pacientes com lombalgia crônica. Rev Dor. 2010;11(2):116-21.

15. Korelo RIG, Ragasson CAP, Lerner CE, Morais JC, Cossa JBN, Karuczuk C. Efeito de um programa cinesioterapêutico de grupo, aliado à escola de postura, na lombalgia crônica. Fisioter Mov. 2013;26(2):389-94.

DOI: http://dx.doi.org/10.1590/s0103-

51502013000200016

Recebido para publicação em 28/08/2015

Revisado em 11/09/2015

Aceito em 15/09/2015 